\title{
Andreev Transport in Double Quantum Dot Cooper Pair Splitters in the Presence of External Magnetic Field
}

\author{
P. TROCHA* AND I. WEYMANN \\ Faculty of Physics, Adam Mickiewicz University, Umultowska 85, 61-614 Poznań, Poland
}

\begin{abstract}
The effect of external magnetic field on the transport properties of double quantum dots coupled to normal and superconducting leads is studied by means of the real-time diagrammatic technique in the sequential tunneling regime. This device works as a gate-controlled Cooper pair splitter. We focus on the transport regime where the current is blocked due to the spin triplet blockade. It is shown that external magnetic field can modify the Andreev current and differential conductance. In particular, magnetic field can suppress the negative differential conductance associated with the triplet blockade.
\end{abstract}

DOI: 10.12693 /APhysPolA.127.502

PACS: 73.23.-b, 73.21.La, 74.45.+c

\section{Introduction}

Transport properties of hybrid quantum dots coupled to superconducting and/or normal leads has recently attracted a lot of attention [1-12]. When the applied bias voltage is smaller than the superconducting energy gap, the normal tunneling processes are suppressed, while the current can flow due to Andreev reflection [13]. In particular, manipulation and control of Cooper paris have recently been demonstrated in double quantum dot (DQD) systems [14-16]. It was shown that DQDs can be used to split Cooper pairs, and such process can be conveniently tuned by gate voltages applied to the dots. Besides the fact that manipulation and splitting of Cooper pairs is exciting from the fundamental point of view, it is also of potential importance for future applications [17].

In this paper we analyze transport properties of DQDs coupled to superconducting and normal leads, focusing on transport regime where the tunneling of Cooper pairs is blocked by enhanced occupation of a triplet state in DQDs. Since Cooper pair involves two electrons of opposite spin, when both dots are occupied by a single electron with the same spin, the Andreev current becomes suppressed [6]. Here, we focus on analyzing the effects of external magnetic field. Our calculations are performed by using the real-time diagrammatic technique including the sequential tunneling processes.

\section{Theoretical description}

The schematic of considered system is shown in Fig. 1. It is based on two capacitively-coupled quantum dots attached to a common $s$-wave BCS superconductor and to two separate metallic electrodes. Since we are interested in the low-bias Andreev current, we assume that the superconducting energy gap is the largest energy scale in

*corresponding author; e-mail: ptrocha@amu.edu.pl the problem and use the following effective Hamiltonian

$$
H=H_{E}+H_{T}+H_{D Q D},
$$

where the first term describes noninteracting electrons in the electrodes, $H_{E}=\sum_{r \boldsymbol{k} \sigma} \varepsilon_{r \boldsymbol{k}} c_{r \boldsymbol{k} \sigma}^{\dagger} c_{r \boldsymbol{k} \sigma}$, with $c_{r \boldsymbol{k} \sigma}^{\dagger}$ creating a spin- $\sigma$ electron with momentum $\boldsymbol{k}$ and energy $\varepsilon_{r \boldsymbol{k}}$ in the left/right $(r=L / R)$ lead. The second term accounts for tunneling processes between DQD and metallic leads

$$
H_{T}=\sum_{r=L, R} \sum_{\boldsymbol{k} \sigma} V_{r}\left(c_{r \boldsymbol{k} \sigma}^{\dagger} d_{r \sigma}+d_{r \sigma}^{\dagger} c_{r \boldsymbol{k} \sigma}\right) \text {. }
$$

Here, $V_{r}$ denotes the tunnel matrix element between the dot $r$ and the corresponding lead, which is assumed to be energy independent, and $d_{r \sigma}^{\dagger}$ is the creation operator for an electron with spin $\sigma$ and energy $\varepsilon_{r \sigma}$ in the dot $r$. The coupling of the dots to external metallic leads gives rise to finite level width, described by, $\Gamma_{r}=2 \pi\left|V_{r}\right|^{2} \rho_{r}$, for dot $r$, where $\rho_{r}$ is the constant density of states of lead $r$. We assume that the couplings are equal, $\Gamma_{L}=\Gamma_{R} \equiv \Gamma / 2$. Furthermore, we assumed that the electrochemical potential of superconducting lead is set to zero, while the potentials of the two normal leads are kept the same and equal to $\mathrm{eV}$.

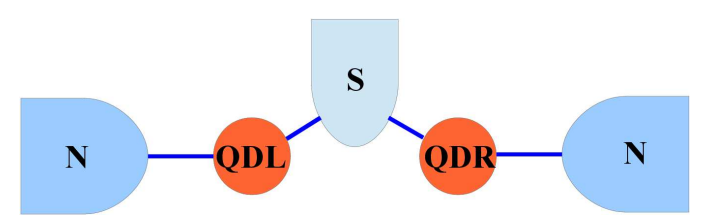

Fig. 1. Schematic of a double quantum dot Cooper pair splitter. Each dot is coupled to its own lead and the two dots are coupled to a common superconducting lead.

Finally, the last term of the Hamiltonian describes the DQD and superconducting lead, in which the superconductor degrees of freedom have been integrated out by assuming infinite energy gap. It has the following form $[6,17]$ 


$$
\begin{aligned}
& H_{D Q D}=\sum_{r=L, R}\left(\sum_{\sigma} \varepsilon_{r \sigma} n_{r \sigma}+U_{r} n_{r \uparrow} n_{r \downarrow}\right) \\
& +\sum_{\sigma \sigma^{\prime}} U n_{L \sigma} n_{R \sigma^{\prime}}+\sum_{r, r^{\prime}=L, R}(-1)^{\delta_{r r^{\prime}}} \frac{\Gamma_{S}}{2} \\
& \times\left(d_{r \uparrow}^{\dagger} d_{r^{\prime} \downarrow}^{\dagger}+d_{r^{\prime} \downarrow} d_{r \uparrow}\right),
\end{aligned}
$$

where $n_{r \sigma}=d_{r \sigma}^{\dagger} d_{r \sigma}, U$ is the Coulomb interaction between the two dots and $U_{r}$ is the Coulomb energy of two electrons residing on the dot $r . \quad \varepsilon_{r \sigma}=\varepsilon+\tilde{\sigma} B / 2$ with $\tilde{\sigma}=1$ for $\sigma=\uparrow$ and $\tilde{\sigma}=-1$ for $\sigma=\downarrow$. Here, $B$ is the external magnetic field expressed in units of $g \mu_{\mathrm{B}}$ and the dot levels are degenerate $\varepsilon_{L}=\varepsilon_{R} \equiv \varepsilon$. The last, particle-nonconserving term describes the creation and annihilation of Cooper pairs in the superconductor, where $\Gamma_{S}$ is the coupling strength to the superconducting lead, assumed to be equal for the two dots.

In this paper we are interested in the transport regime where the Cooper pairs are split and the electrons tunnel through the two dots to separate leads. Such processes are called crossed Andreev reflection. To exclude the processes where the Cooper pair enters the same lead (direct Andreev reflection), we set the Coulomb energy on each dot to infinity to exclude double occupancy of each dot, $U_{L}, U_{R} \rightarrow \infty$. Then, the double dot is described by 9 states, $|\chi\rangle$, which are the many-body eigenstates of the Hamiltonian (3). The occupation probabilities of those states and the tunneling current $I_{r}$ flowing through the junction $r$ between given dot and metallic lead can be found by using the real-time diagrammatic technique [19-21]. This technique is based upon a systematic perturbation expansion with respect to the coupling strength $\Gamma$. Here we consider the lowest order of expansion, which corresponds to sequential tunneling processes. Knowing the currents $I_{L}$ and $I_{R}$, one can calculate the current flowing between the DQD and superconductor from the Kirchhoff's law, $I_{S}=I_{L}+I_{R}$.

The Andreev current $I_{S}$ and the respective differential conductance $G_{S}=\mathrm{d} I_{S} / \mathrm{d} V$ are shown in Fig. 2 as a function of bias voltage applied to the two normal leads. This figure is calculated for zero detuning of the levels $\delta=2 \varepsilon+U=0$ and for different magnetic fields, as indicated in the figure. In the case of $\delta=0$, i.e. when the empty and doubly occupied (with one electron on each dot) states are degenerate, the Andreev current can be maximized to reach $I_{S}=I_{0}=e \Gamma / \hbar$, cf. Fig. 2a. First of all, one can note that in the absence of magnetic field and for negative bias voltage, the Andreev current exhibits typical Coulomb staircase, see Fig. 2a, associated with consecutive bound states that start taking part in transport with increasing the bias voltage. At a voltage corresponding to a step in the current, the differential conductance exhibits a peak, Fig. 2b. On the other hand, for positive bias voltage, the current increases and then suddenly drops to zero. This is due to the fact that for $e V / U \gtrsim 1 / 2$, the double dot becomes trapped in a spin triplet state. Since injecting or extracting Cooper pairs to/from superconductor involves spin-up and spin-

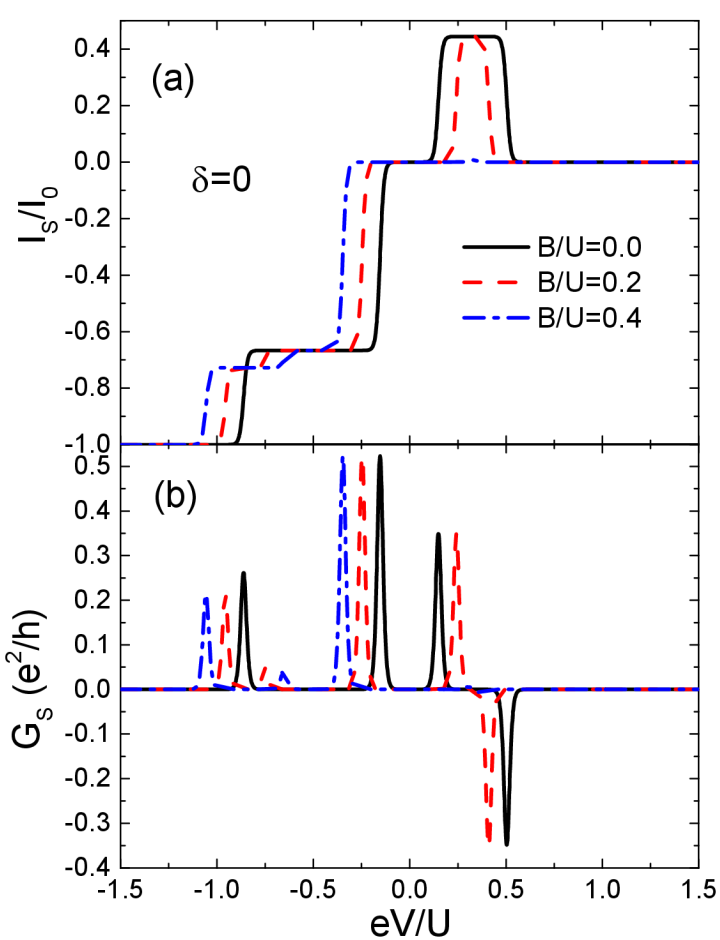

Fig. 2. The Andreev current $I_{S}$ (a) and the corresponding differential conductance $G_{S}$ (b) as a function of bias voltage calculated for different magnetic fields, as indicated. The other parameters are: $U=1$ (used as energy unit), $\delta=2 \varepsilon+U=0, \Gamma_{S}=0.5, \Gamma=0.01$ and $T=0.01$, with $I_{0}=e \hbar / \Gamma$.

down electrons, full occupation of triplet state blocks the Andreev current flowing through the device. This also leads to corresponding negative differential conductance, which is clearly visible in Fig. 2 b.

In the case of finite magnetic field, the Andreev bound states can become split and, because of that, the currentvoltage characteristics exhibit more steps, see e.g. Fig. 2 for $B / U=0.2$ and $B / U=0.4$. Moreover, one can see that the width of the first plateau in the current for positive voltage becomes decreased with increasing $B$. An interesting situation occurs for $B / U=0.4$, when $I_{S}$ becomes suppressed for whole range of positive bias voltage considered in this figure. Consequently, the effect of negative differential conductance is almost absent. More specifically, finite magnetic field changes the range of bias voltage for which the triplet blockade occurs, such that for $B / U=0.4$ the blockade is extended to the whole range of $e V / U \gtrsim 0.25$, see Fig. 2a, (note that the range around $e V=0$ still corresponds to the Coulomb blockade regime).

When the detuning of DQD levels is present, the Andreev processes become generally decreased. This is because now there is a difference between the energies of empty and doubly occupied states and it is more difficult to form a Cooper pair. The corresponding current and differential conductance are shown in Fig. 3 for $\delta=1$. 
Also in this case one can see the blockade of the current due to occupation of the triplet state, which is now visible for $e V / U>1$ in the absence of $B$. For negative bias voltage, on the other hand, the current and associated $\mathrm{d} I_{S} / \mathrm{d} V$ behave in a rather typical way, with $I_{S}$ varying monotonously with $\mathrm{eV}$. The effect of finite magnetic field is here clearly visible. First, the region of the triplet blockade is shifted towards smaller bias voltages with increasing the magnetic field, see Fig. 3a. Second, magnetic field splits the levels and additional steps in the current are present for negative bias. Correspondingly, the differential conductance displays then additional peaks, separation of which increases with raising the magnetic field, see Fig. 3b.

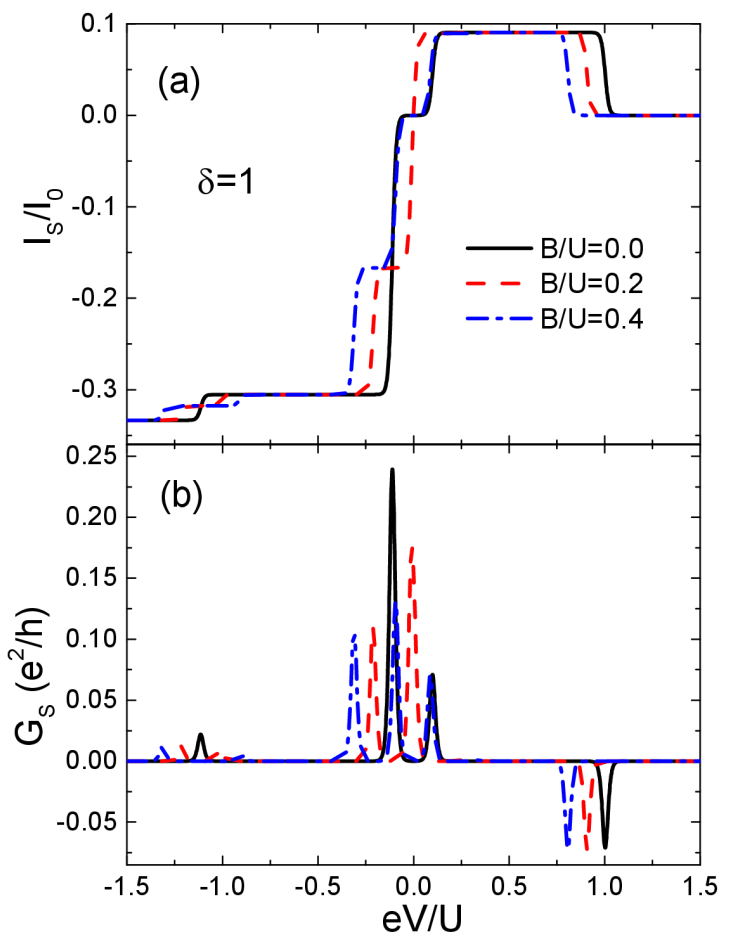

Fig. 3. The same as in Fig. 2 calculated for $\delta=1$.

\section{Conclusions}

In this paper we have analyzed the Andreev current flowing in double quantum dot based Cooper pair splitters in the presence of external magnetic field. The current flowing through such device exhibits an asymmetry with respect to the bias reversal and becomes suppressed for one bias polarization due to enhanced occupation of the triplet state. We have shown that magnetic field changes the range of bias voltage for which the blockade occurs. Moreover, it can also suppress the effect of negative differential conductance associated with the triplet blockade.

\section{Acknowledgments}

This work was supported from the 'Iuventus Plus' project No. IP2011 059471 for years 2012-2014.

\section{References}

[1] A. Martin-Rodero, A. Levy Yeyati, Adv. Phys. 60, 899 (2011).

[2] D. Beckmann, H.B. Weber, H. v. Löhneysen, Phys. Rev. Lett. 93, 197003 (2004).

[3] S. Russo, M. Kroug, T.M. Klapwijk, A.F. Morpurgo, Phys. Rev. Lett. 95, 027002 (2005).

[4] D. Futterer, M. Governale, M. G. Pala, J. König, Phys. Rev. B 79, 054505 (2009).

[5] B. Sothmann, D. Futterer, M. Governale, J. König, Phys. Rev. B 82, 094514 (2010).

[6] J. Eldridge, M.G. Pala, M. Governale, J. König, Phys. Rev. B 82, 184507 (2010).

[7] L. Hofstetter, A. Geresdi, M. Aagesen, J. Nygård, C. Schönenberger, S. Csonka, Phys. Rev. Lett. 104, 246804 (2010).

[8] D. Chevallier, J. Rech, T. Jonckheere, T. Martin, Phys. Rev. B 83, 125421 (2011).

[9] J. Rech, D. Chevallier, T. Jonckheere, T. Martin, Phys. Rev. B 85, 035419 (2012).

[10] K.P. Wójcik, I. Weymann, Phys. Rev. B 89, 165303 (2014).

[11] I. Weymann, P. Trocha, Phys. Rev. B 89, 115305 (2014).

[12] P. Trocha, J. Barnaś, Phys. Rev. B 89, 245418 (2014).

[13] A.F. Andreev, Zh. Eksp. Teor. Fiz. 46, 1823 (1964) [ Sov. Phys. JETP 19, 1228 (1964)].

[14] L. Hofstetter, S. Csonka, J. Nygård, C. Schönenberger, Nature 461, 960 (2010).

[15] L.G. Herrmann, F. Portier, P. Roche, A. Levy Yeyati, T. Kontos, C. Strunk, Phys. Rev. Lett. 104, 026801 (2010).

[16] L. Hofstetter, S. Csonka, A. Baumgartner, G. Fülöp, S. d'Hollosy, J. Nygård, C. Schönenberger, Phys. Rev. Lett. 107, 136801 (2011).

[17] S. de Franceschi, L.P. Kouwenhoven, C. Schönenberger, W. Wernsdorfer, Nat. Nanotech. 5, 703 (2011).

[18] A.V. Rozhkov, D.P. Arovas, Phys. Rev. B 62, 6687 (2000).

[19] H. Schoeller, G. Schön, Phys. Rev. B 50, 18436 (1994); J. König, J. Schmid, H. Schoeller, G. Schön, Phys. Rev. B 54, 16820 (1996).

[20] M. Governale, M. G. Pala, J. König, Phys. Rev. B 77, 134513 (2008).

[21] I. Weymann, Phys. Rev. B 78, 045310 (2008). 